\title{
ON MODULES OF CONTINUOUS LINEAR MAPPINGS
}

\author{
DINAMÉRICO P. POMBO JR. \\ Instituto de Matemátıca \\ Universidade Federal Fluminense \\ Rua São Paulo, s/no \\ 24020-005 Niteróı, RJ, BRASIL
}

(Recelved April 4, 1996)

\begin{abstract}
Modules of contınuous linear mappings with values in topological modules of contunuous mappings are studied
\end{abstract}

KEY WORDS AND PHRASES: Topological modules, contınuous linear mappings, contunuous mappings. 1991 AMS SUBJECT CLASSIFICATION CODE: $46 \mathrm{H} 25$

\section{INTRODUCTION}

Grothendieck [1] has proved that, under certain conditions, spaces of continuous linear mappings on locally convex spaces are naturally isomorphic to spaces of contunuous mappings on locally compact topological spaces, either as vector spaces or even as locally convex spaces Non-linear versions of his results have been discussed in [2]

In the present note, we use Grothendieck's argument to show that modules of continuous linear mappings with values in topological modules of continuous mappings may be identified with modules of continuous mappings with values in topological modules of continuous linear forms.

Throughout this note, $A$ denotes a commutative topological ning with an identity element and all $A$-modules under consideration are unitary $T$ represents an arbitrary topological space, $\mathcal{C}(T ; S)$ the $A$-module of all contınuous mappings from $T$ into the topological $A$-module $S$ and $\mathcal{C}(T):=\mathcal{C}(T ; A)$. Given two topological $A$ modules $R$ and $S, \mathcal{L}(R ; S)$ represents the $A$-module of all continuous $A$-linear mappings from $R$ into $S$ and $R^{\prime}:=\mathcal{L}(R ; A)$. Moreover, $R_{s}^{\prime}$ denotes the $A$-module $R^{\prime}$ endowed with the $A$-module topology $\tau_{s}$ of pointwre convergence.

PROPOSITION 1. Let $E, F$ be topological $A$-modules and $u \in \mathcal{L}(E ; F)$ Then ${ }^{t} u \in \mathcal{L}\left(F_{s}^{\prime} ; E_{s}^{\prime}\right)$ and ${ }^{t} u$ transforms equicontinuous subsets of $F^{\prime}$ into equicontinuous subsets of $E^{\prime}$, where ${ }^{t} u$ is the adjoint of $u$

PROOF. Straightforward.

Consider $\mathcal{C}(T)$ endowed with the topology of compact convergence. By Theorem 154 (1) of [5] and Proposition (a) of [3], $\mathcal{C}(T)$ is a topological $A$-module. (The proposition just mentioned will also ensure that other function spaces which will appear in the sequel are topological $A$-modules.)

For each $t \in T$, let $\delta(t): \mathcal{C}(T) \rightarrow A$ be given by $\delta(t)(f)=f(t)$ for $f \in \mathcal{C}(T)$. Then $\delta(t) \in(\mathcal{C}(T))^{\prime}$

PROPOSITION 2. The mapping

$$
\delta: t \in T \mapsto \delta(t) \in(\mathcal{C}(T))^{\prime}
$$

is continuous when $(\mathcal{C}(T))^{\prime}$ is endowed with $\tau_{s}$, and transforms compact subsets of $T$ into equicontunuous subsets of $(\mathcal{C}(T))^{\prime}$

PROOF. Let $t_{0} \in T, f \in \mathcal{C}(T)$ and $W$ a neighborhood of zero in $A$. By the contunuity of $f$ at $t_{0}$, there exists a neighborhood $V$ of $t_{0}$ in $T$ such that $f(t)-f\left(t_{0}\right) \in W$ for all $t \in V$, that is, $\left(\delta(t)-\delta\left(t_{0}\right)\right)(f) \in W$ for all $t \in V$. This proves the contınuity of $\delta$ at $t_{0}$. Moreover, if $K$ is a compact subset of $T$, then

$$
\delta(K)(\{f \in \mathcal{C}(T) ; f(K) \subset W\}) \subset W .
$$


This shows that $\delta(K)$ is equicontinuous, thereby concluding the proof.

Let $E$ be a topological $A$-module For each $u \in \mathcal{L}(E ; \mathcal{C}(T))$, define $\Psi(u)={ }^{t} u \circ \delta$ By Propositions 1 and 2, $\Psi(u) \in \mathcal{C}\left(T ; E_{s}^{\prime}\right)$ and $\Psi(u)$ transforms compact subsets of $T$ into equicontinuous subsets of $E^{\prime}$ Let $\Psi$ be the $A$-linear mapping

$$
u \in \mathcal{L}(E ; \mathcal{C}(T)) \mapsto \Psi(u) \in \mathcal{C}\left(T ; E_{s}^{\prime}\right),
$$

and let $H$ be the submodule of $\mathcal{C}\left(T ; E_{s}^{\prime}\right)$ formed by the continuous mappings $h: T \rightarrow E_{s}^{\prime}$ such that $h(K)$ is an equicontinuous subset of $E^{\prime}$ for every compact subset $K$ of $T$.

THEOREM. $\Psi$ is an $A$-module isomorphism between $\mathcal{L}(E ; \mathcal{C}(T))$ and $H$

PROOF. We have just observed that $\operatorname{Im}(\Psi) \subset H$.

We clam that $\Psi$ is injective. Indeed, take a $u \in \mathcal{L}(E ; \mathcal{C}(T))$ such that $\Psi(u)=0$, and fix an $x \in E$ Then, for all $t \in T$,

$$
\left[\left({ }^{t} u \circ \delta\right)(t)\right](x)=[\delta(t) \circ u](x)=u(x)(t)=0 .
$$

Therefore $u(x)=0$, and so $u=0$.

Now, let us verify that $H \subset \operatorname{Im}(\Psi)$ Indeed, if $h \in H$, define $u(x)(t)=h(t)(x)$ for $x \in E, t \in T$. Then $u(x) \in \mathcal{C}(T)$ since $u(x)=\Phi \circ h$, where $\Phi$ is the $\tau_{s}$-continuous $A$-linear mapping $\varphi \in E^{\prime} \mapsto \varphi(x) \in A$. Moreover, it is easily seen that $u$ is an $A$-linear mapping from $E$ into $\mathcal{C}(T)$ In order to prove the continuty of $u$, let $K$ be a compact subset of $T$ and $W$ a neighborhood of zero in $A$ By the equicontinuity of $h(K)$, there exists a neighborhood $U$ of zero in $E$ such that $h(K)(U) \subset W$. Therefore

$$
u(U) \subset\{f \in \mathcal{C}(T) ; f(K) \subset W\},
$$

which proves the continuity of $u$. Finally, it is clear that $\Psi(u)=h$, which concludes the proof of the theorem.

Let $E$ be a topological $A$-module. Let $\mathcal{M}$ be a family of bounded subsets of $E$ such that for every $B_{1}, B_{2} \in \mathcal{M}$ there is a $B_{3} \in \mathcal{M}$ with $B_{1} \cup B_{2} \subset B_{3}$, and let $\tau_{\mathcal{M}}$ be the $A$-module topology on $E^{\prime}$ of $\mathcal{M}$ convergence. By Theorem 15.2 (1), (4) of [5], the set $G$ of all mappings $g: T \rightarrow E^{\prime}$ such that $g(K)$ is $\tau_{\mathcal{M}^{-}}$ bounded in $E^{\prime}$ for every compact subset $K$ of $T$ is an $A$-module. By Theorem 25.5 of [5], $H \subset G$, and hence $H$ is a submodule of $G$. Consider $E^{\prime}$ endowed with $\tau_{\mathcal{M}}$ Then $G$, endowed with the topology of compact convergence, is a topological $A$-module. Consider on $H$ the $A$-module topology induced by that of $G$

COROLLARY 1. $\Psi$ is a topological $A$-module isomorphism between $\mathcal{L}_{\mathcal{M}}(E ; \mathcal{C}(T))$ and $H$, where $\mathcal{L}_{\mathcal{M}}(E ; \mathcal{C}(T))$ denotes $\mathcal{L}(E ; \mathcal{C}(T))$ endowed with the $A$-module topology of $\mathcal{M}$-convergence.

PROOF. It suffices to observe that, if $B \in \mathcal{M}, K$ is a compact subset of $T$ and $W$ is a neighborhood of zero in $A$, then $u \in\{v \in \mathcal{L}(E ; \mathcal{C}(T)) ; v(B)(K) \subset W\}$ (a basic neighborhood of zero in $\mathcal{L}_{\mathcal{M}}(E ; \mathcal{C}(T)$ )) if and only if $\Psi(u) \in\{h \in H ; h(K)(B) \subset W\}$ (a basic neighborhood of zero in $H$ ).

If $E$ is a barrelled topological $A$-module ([4], Definition 2.1), Theorem 15.4 (1) of [5] and Theorem 31 of [4] imply that $H=\mathcal{C}\left(T ; E_{s}^{\prime}\right)$ As a consequence, Corollary 1 yields:

COROLLARY 2. If $E$ is a barrelled topological $A$-module, then $\mathcal{L}_{\mathcal{M}}(E ; \mathcal{C}(T))$ and $\mathcal{C}\left(T ; E_{s}^{\prime}\right)$ are isomorphic as topological $A$-modules.

\section{REFERENCES}

[1] GROTHENDIECK, A., Espaces Vectoriels Topologiques, 3rd ed., Publ. Soc. Mat. São Paulo (1964)

[2] POMBO, D.P., Jr., On adjoints of non-linear mappings, Bull. Austral. Math. Soc 33 (1986), 227-236

[3] POMBO, D.P., Jr., On the completion of certain topological modules, Math. Japonica 37 (1992), 333-336.

[4] POMBO, D.P., Jr., On barrelled topological modules, Internat. J. Math. \& Math. Scl. 19 (1996), 45-52

[5] WARNER, S., Topological Fields, Notas de Matemática No. 126, North-Holland Publishing Company (1989). 


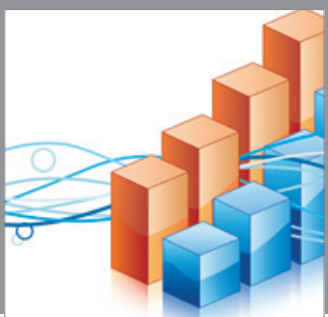

Advances in

Operations Research

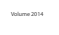

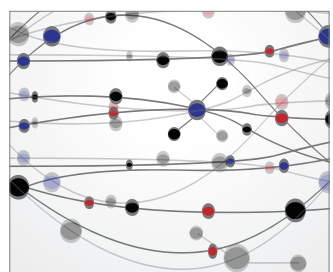

\section{The Scientific} World Journal
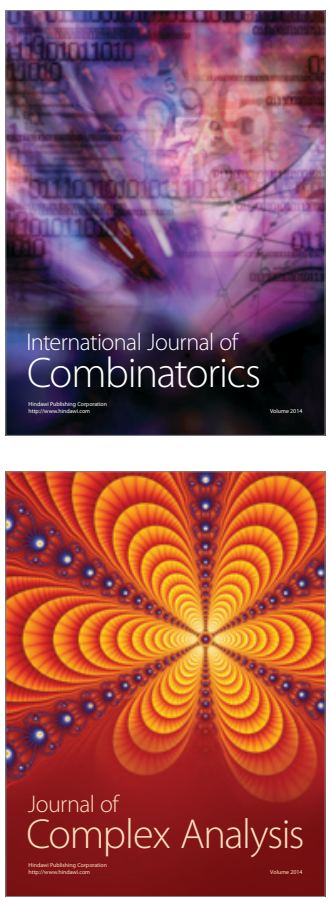

International Journal of

Mathematics and

Mathematical

Sciences
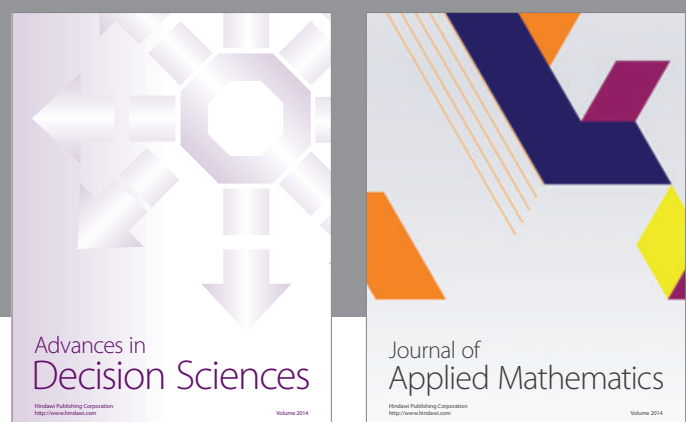

Journal of

Applied Mathematics
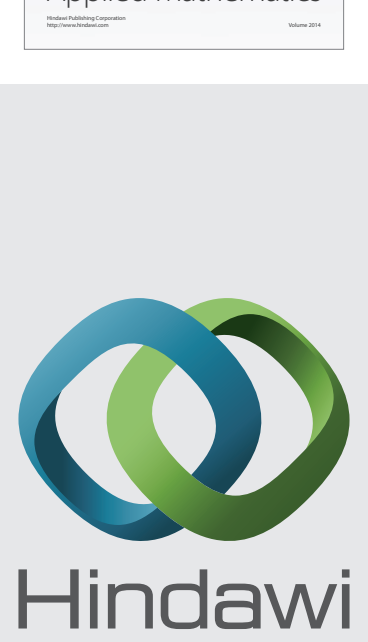

Submit your manuscripts at http://www.hindawi.com
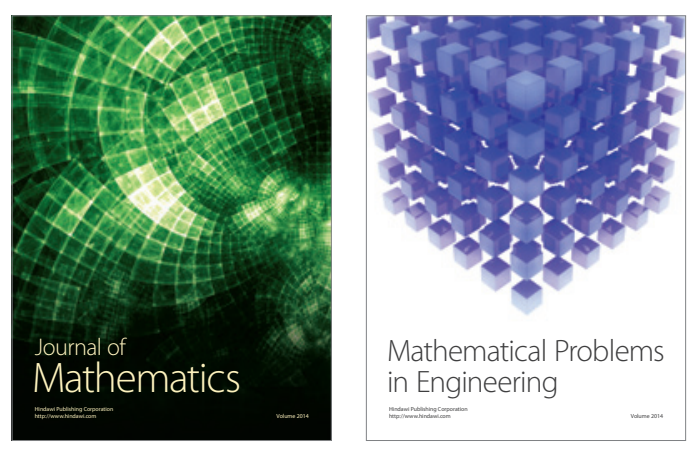

Mathematical Problems in Engineering
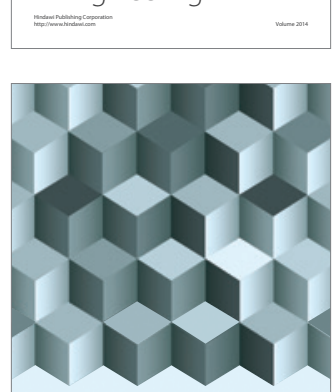

Journal of

Function Spaces
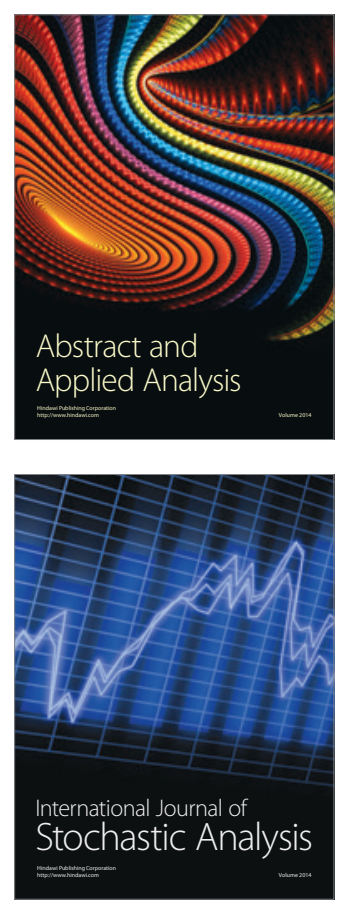

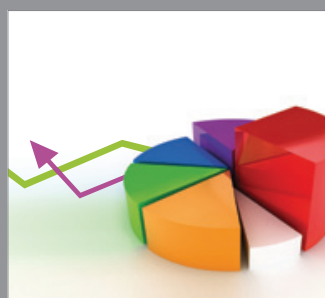

ournal of

Probability and Statistics

Promensencen
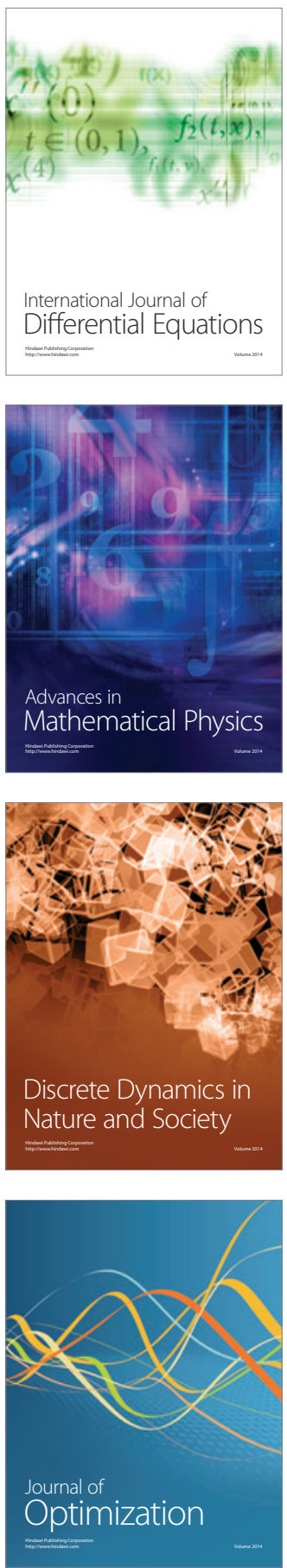